\title{
Flimmert der Vorhof, schützt Apixaban das Hirn
}

\begin{abstract}
Nicht jeder Patient mit Vorhofflimmern, bei dem ein erhöhtes Schlaganfallrisiko besteht, kann oder will eine orale Antikoagulation mit Vitamin-K-Antagonisten zur Prävention bekommen. Für diese Gruppe könnte bald eine neue Option verfügbar sein, die Experten als „Durchbruch in der modernen Schlaganfallprävention" begrüßen.
\end{abstract}

Viele Patienten mit Vorhofflimmern bekommen den Thrombozytenhemmer Acetylsalicylsäure (ASS) zur Prävention von Schlaganfällen. Das könnte demnächst anders werden: In der AVERROES-Studie hat sich der neue Gerinnungshemmer Apixaban in der Verhinderung von Schlaganfällen im Vergleich zu ASS als wesentlich effektiver erwiesen. „Dieses Ergebnis ist ein Durchbruch in der modernen Schlaganfallprävention und wird die Weichen in der Behandlung völlig neu stellen", urteilt der Essener Neurologe Professor Hans Christoph Diener in einer aktuellen Pressemitteilung der Deutschen Gesellschaft für Neurologie (DGN) und der Deutschen Schlaganfall-Gesellschaft.

Vitamin-K-Antagonisten können das Schlaganfallrisiko bei Vorhofflimmern um $70-80 \%$ senken. Viele Patienten, die geeignete Kandidaten für eine orale Antikoagulation mit Vitamin-K-Antagonisten sind, erhalten aber in der Praxis de facto keine entsprechende Prophylaxe.

In den letzten Jahren sind neue Gerinnungshemmer entwickelt worden, die im Vergleich zu Vitamin-K-Antagonisten Vorteile aufweisen: Sie können in fixer Dosierung verabreicht werden und führen zu einer zuverlässigen Hemmung der
Blutgerinnung, ohne dass regelmäßige Gerinnungskontrollen notwendig sind.

\section{Studie wurde vorzeitig beendet}

Einer dieser neuen Wirkstoffe ist der Faktor-Xa-Hemmer Apixaban, dessen klinische Entwicklung von den Unternehmen Bristol-Myers Squibb und Pfizer in einem umfangreichen Forschungsprogramm betrieben wird. Teil dieses Programms ist die AVERROES-Studie. In diese Studie sind 5599 Patienten mit Vorhofflimmern und erhöhtem Schlaganfallrisiko aufgenommen worden, die entweder Vitamin-K-Antagonisten nicht einnehmen wollten oder nach Einschätzung des behandelnden Arztes ungeeignet für eine solche Prophylaxe waren.

Die Studienteilnehmer sind randomisiert einer Prophylaxe mit Apixaban (Zieldosis: $5 \mathrm{mg}$, zweimal täglich) oder dem Plättchenhemmer ASS (81-324 mg/Tag) zugeteilt worden. Primäres Zielkriterium war das Auftreten von Schlaganfällen oder systemischen Embolien.

Schon eine Zwischenanalyse offenbarte einen deutlichen Unterschied bei der Ereignisrate zugunsten der Apixaban-Gruppe im Vergleich zur ASSGruppe. Daraufhin wurde die Studie nach einer mittleren Beobachtungsdauer von 1,1 Jahren vorzeitig beendet.

Die jährliche Rate von Schlaganfälle und systemischen Embolien betrug demnach 1,6\% (Apixaban) und 3,7\% (ASS), was einer signifikanten relativen Risikoreduktion um 55\% durch den Faktor-Xa-Hemmer entspricht (51 versus 113 Ereignisse). Die Mortalitätsraten in beiden Gruppen unterschieden sich nicht signifikant (3,5 versus 4,4\%). Das Risiko einer Klinikeinweisung aus kardiovaskulären Gründen war in der Apixaban-Gruppe signifikant um $21 \%$ niedriger (12,6 versus $15,9 \%)$.

\section{Wie ist der absolute Nutzen?}

Die Rate schwerer Blutungen war in der Apixaban-Gruppe nur leicht und nicht signifikant erhöht (1,4 versus 1,2\%). Die Zahl der intrakraniellen Blutungen war in beiden Gruppen nahezu gleich. Apixaban führte seltener zu Therapieabbrüchen als ASS.

Der absolute Nutzen von Apixaban lässt sich auf Basis der Studiendaten so beziffern: Werden 1000 Patienten mit Vorhofflimmern und erhöhtem Schlaganfallrisiko ein Jahr lang mit Apixaban statt mit ASS behandelt, lassen sich zusätzlich 21 Schlaganfälle oder systemische Embolien, neun Todesfälle und 33 kardiovaskulär bedingte Klinikeinweisungen verhindern, für den Preis von zwei schwerwiegenden Blutungskomplikationen.

(Peter Overbeck)

N Engl J Med 2011, 364:806 